\title{
Surface Photometry of Barred AGN Arakelian 564
}

\author{
G. Petrov, L. Slavcheva, R. Bachev, B. Mihov \\ Institute for Astronomy, Bulgarian Academy of Sciences, Bulgaria
}

The Seyfert 1.5 galaxy Akn564 [1] is a well known X-ray active galactic nucleus (AGN) included in our list of selected barred AGN. The galaxy was observed during the August 1996 season at the 2-m RCC telescope of the Astronomical Observatory "Rozhen" of the Bulgarian Academy of Sciences. ST-6 with standard Schott V, R, I filters were used. MIDAS'96 package was used for the data reduction with teh Richter's expansion for the surface photometry [2]. Most of the basic data for the galaxy are shown in the Table 1 below.

Table 1. Observational data for Sy1.5 galaxy Arakelian 564

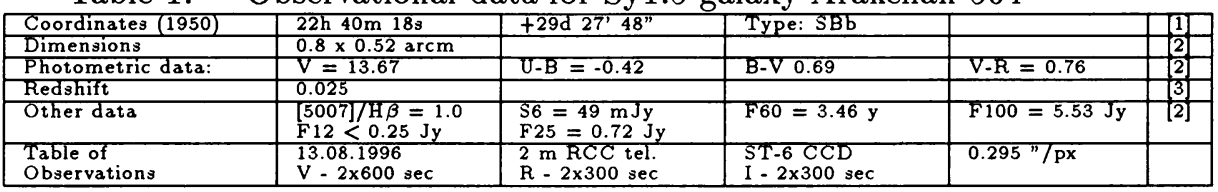

References to the Table: [1] Arakelian, M.A., 1975, Publ.Bjurak.Obs, 47,3. [2] Lipovettsky, V.A. et al., 1987, Comm. of SAO (UdSSR), 55, 5. [3] Arakelian, M.A. et al., 1976, Astrophysics, 12, 456.

Following Richter, flat-fielded, dark-subtracted CCD frames were adaptive filtered, and a multiple masking technique was applied to have "galaxy on a pure background." The real objects were modeled with ellipses according to Bender \& Moelenhoff [3] methods. Cumulative magnitudes and surface brightness for V,R and I frames and for the models have been evaluated, as well as the distribution of the position angles of the major axes and the colors. The parameters of the disk in $3-18$ arcsec radius for the objects and models in the three colors are presented in Table 2.

Here $\mathrm{SB}=\mathrm{Mc}+(\mathrm{r} / \mathrm{rc}) \exp (1 / \mathrm{n})$. On figure 1 (only $\mathrm{R}$ color is shown) the twisted bar, wider and stronger in the south part, is clearly seen and some bright star formation regions are traced. The extremely bright starlike nucleus with diameters $8-10$ arcsec includes about $60 \%$ of the luminosity of the galaxy. The disk is clearly visible to 50 arcsec on $\mathrm{SB}=26 \mathrm{mag} / \mathrm{sqr}$.arcsec. The nucleus is bigger in $\mathrm{R}$ and I colors - see the sharp minimum in the distribution of V-R colors for the object and model. Figure 2 presents the surface brightness (SB), color index (V-R), axis ratio (b/a) and position angle (PA) as a function of the radius. 
Table 2. Akn564 Fitting parameters

\begin{tabular}{|l|l|l|l|l|l|}
\hline mod_obj & $\mathrm{n}$ & $\mathrm{mc}$ & err_mc & rc & err_rc \\
\hline & & & & & \\
\hline SB_v_mod & +0.40 & 21.36 & 0.782 & +0.0029 & +.00026 \\
\hline SB_r_mod & +0.40 & 21.94 & 0.168 & +0.0018 & +.00005 \\
\hline SB_i_mod & +0.40 & +19.81 & 2.030 & +0.0042 & +.00036 \\
\hline & & & & & \\
\hline SB_v_obj & +0.40 & +20.37 & +0.780 & +0.0039 & +.00029 \\
\hline SB_r_obj & +0.40 & +20.31 & +0.732 & +0.0032 & +.00019 \\
\hline SB_i_obj & +0.90 & +18.92 & +0.391 & +0.1298 & +.00718 \\
\hline
\end{tabular}

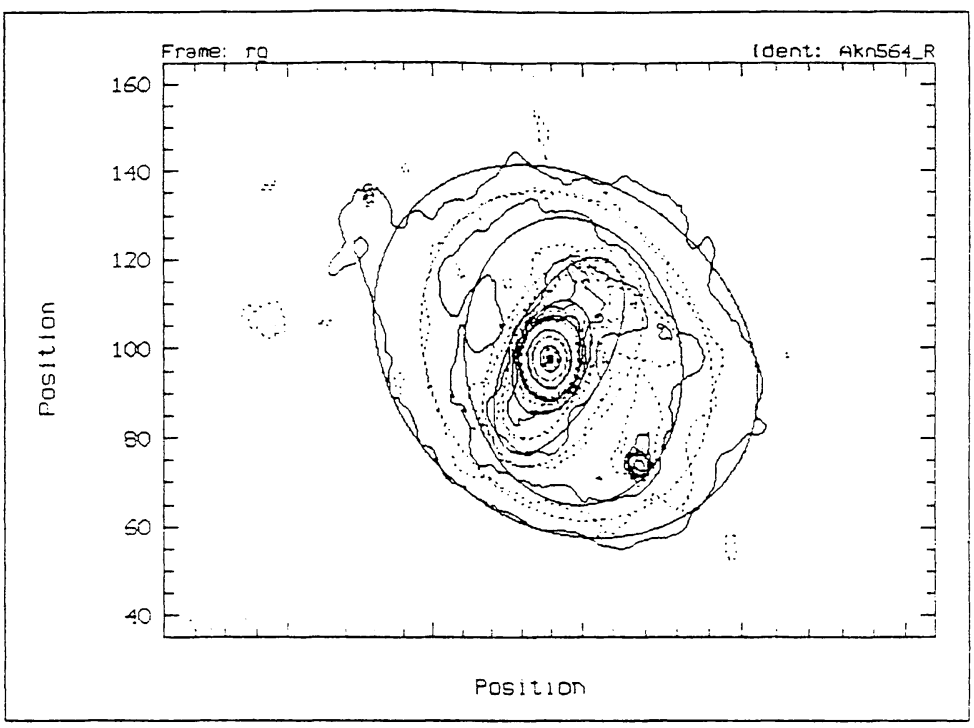

Figure 1. Fitting of the R-frame of Akn 564 with ellipses according to [4] 


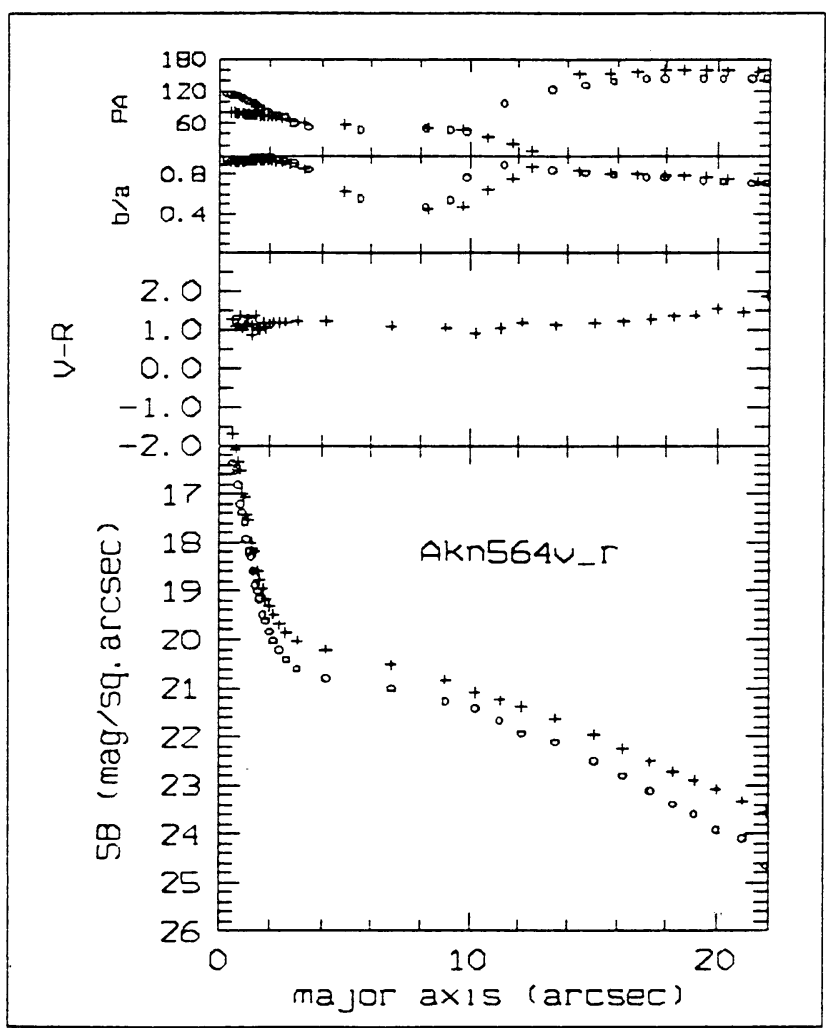

Figure 2. Surface brightness (SB), color index (V-R), axis ratio (b/a) and position angle (PA) as a function of the radius.

\section{References}

Arakelian, M.A., 1975, Publ.Bjurak.Obs., 47, 3

Vennik, J., Hopp, U., Kovachev, B., Kuhn, B., Elsasser, H., 1996, AApSuppl., 117,261

Lorenz, H., Richter, G., Cappaccioli, M., Longo, G., 1993, A\&A, 277, 321

Bender, R., Moelenhoff, C., 1987, A\&A, 177, 71 